\title{
Parallel-tracking bottom-up approaches within chronic disease prevention programmes
}

\author{
Glenn Laverack
}

Published online: 13 September 2011

(c) Swiss School of Public Health 2011

\section{Introduction}

The purpose of this commentary is to discuss how to accommodate top-down and bottom-up approaches at each phase of chronic disease prevention programmes. The commentary uses a theoretical example of targeting a high risk group in New Zealand.

Chronic disease prevention programmes are typically designed to address changes in lifestyle and behaviour centred on, for example, diabetes prevention. There is evidence to suggest that lifestyle and behaviour programmes that include multi-risk factor interventions such as increasing physical activity, dietary modifications and weight loss can significantly reduce the incidence of diabetes in persons at high risk (Knowler et al. 2002; Tuomilehto et al. 2001). In practice, chronic disease prevention programmes are most commonly implemented as activities set within the context of a programme. Professionally led, it is the practitioner or their agency that chooses the design, the means of implementation and evaluation of the programme. This includes the selection of 'targeted groups' and the methods to be used to reach them. The issues to be addressed are traditionally based on epidemiological evidence, rather than on the concerns of the individuals and communities to whom it should concern. This style of programming is called 'top-down'. Bottom-up programmes are fewer in design and address the concerns of the beneficiaries based on discussions with them prior to implementation. The concerns are prioritised and then developed into a form that makes sense to all the programme stakeholders (Laverack and Labonte 2000).

G. Laverack $(\bowtie)$

41 Algarth Road, Pocklington, UK

e-mail: Grlavera@hotmail.com
It is important to remember that the terms 'top-down' and 'bottom-up' are ideal types of practice. 'Top-down' describes programmes where problem identification comes from those in top structures 'down' to the community. 'Bottom-up' is the reverse, where the community identifies its own problems and communicates these to those who have the decision making authority. The practitioner will try to use their influence to 'push-down' a predefined agenda onto the community through top-down programming. The community attempts to 'push-up' an agenda based on their immediate concerns that are often not the same as those identified by the practitioner. The failure of top-down programming to accommodate bottom-up concerns may have a detrimental effect on outcomes. For example, the modest success of top-down programming has only been with higher socio-economic groups: between 1998 and 2004 there was a 9\% decrease in smoking in the lowest quintile in Australia compared to a $35 \%$ decrease in the highest quintile (Baum 2007). This is because top-down programming uses strategies that are effective at reaching the educated and self-motivated sectors of society and are not specifically tailored for low socio-economic or marginalised groups.

The challenge is how to accommodate bottom-up approaches within the dominant top-down styles of programming. This requires a fundamental change in the way we think about chronic disease prevention programmes. Rather than viewing the issue as a bottom-up versus topdown tension, accommodating both can be better viewed as a 'parallel track'. The tensions between the two then occur at each stage of the programme cycle making their resolution much easier to achieve (Laverack 2004). The main purpose of the programme remains unchanged with a focus on the more conventional top-down issues and so fits within the expectations of, and is therefore acceptable to, 
funding agencies but still has a clearly defined role to address community needs and to build capacity.

\section{Accommodating bottom-up approaches}

In Auckland, New Zealand, Polynesian people aged 45 years and above have rates for cardiovascular heart disease which are consistently and significantly higher (about twice as high) than those in the total population. Polynesian males and females also have higher prevalence rates for diabetes and worse causal related indicators for obesity, diet, physical exercise and tobacco consumption (Ministry of health and Pacific Island Affairs 2004). The following is a brief description of how to combine a top-down chronic disease prevention programme and a bottom-up approach through parallel-tracking (see Table 1) targeting Polynesian people.

The programme design phase

It is at the design phase that the relationship is established between the practitioner, or their agency, and the intended beneficiaries. 'Top-down' is a form of authority in which

Table 1 Parallel-tracking bottom-up and top-down approaches

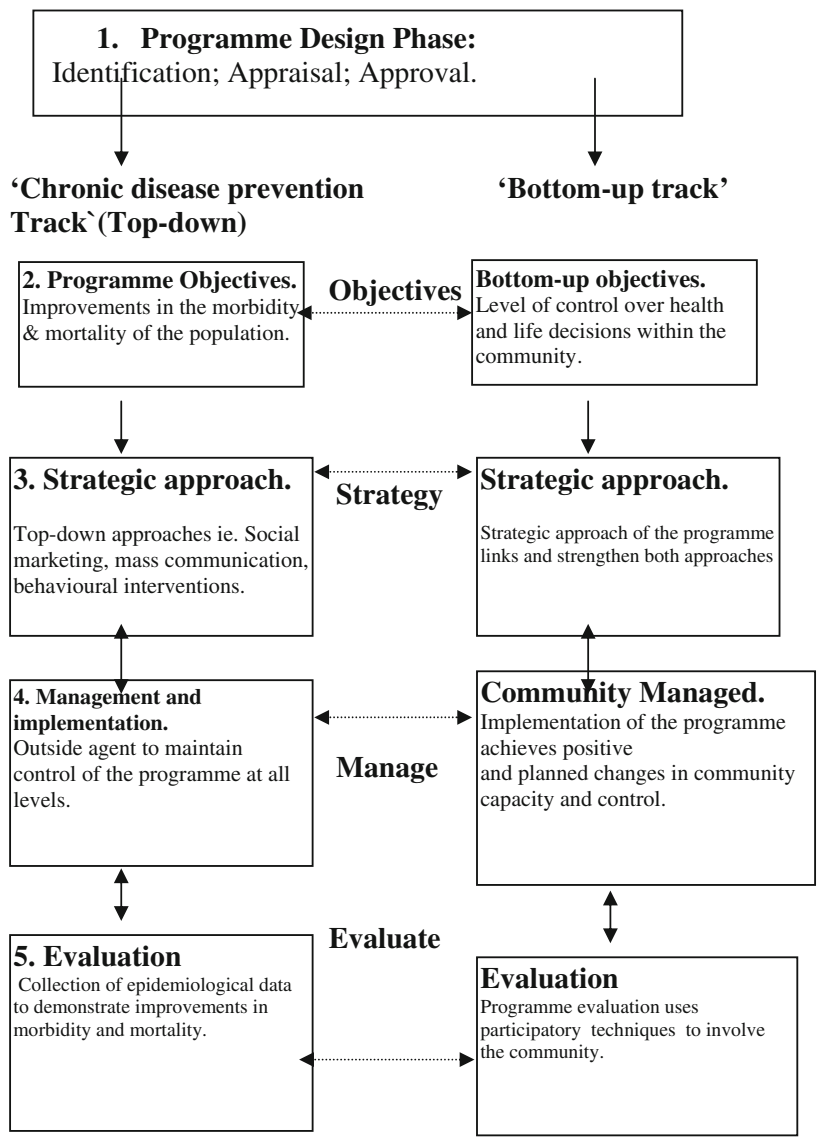

control has traditionally been exerted and facilitated through the programme design. Parallel-tracking moves the practitioner to a position in which control is shared by all stakeholders. It provides a more precise role for the practitioner, one that helps the community to gain control over concerns that influence their lives and health. At the design phase, the practitioner facilitates the involvement of the community members by identifying with them the issues that will be addressed through the programme. The purpose is to actively engage with the community representatives to give them a voice to express their concerns.

With regard to chronic disease in Polynesian peoples these concerns have been identified by Tongan communities in Auckland, New Zealand as (Moana 2005):

- Better facilities for vegetable gardening. A good form of exercise and an activity that people wanted to do, especially the aged;

- Walking groups for women. A means of also building social networks and support groups based around a physical activity;

- More information about diabetes. In both English and Polynesian languages presented through appropriate channels of communication.

The main programme track defines the disease prevention issues to be addressed as:

- Reduce obesity/abdominal fat determined through body mass index;

- Reduce hypertension;

- Reduce high cholesterol levels.

The issue at stake is how the 'chronic disease prevention track' (defined by the outside agency) and the 'bottom-up track' (defined by the community) can become linked during the progressive stages of the programme. The purpose is to achieve the disease prevention objectives and also to build capacity to support the development of the community. Community capacity building is central to the bottom-up approach and involves the development of community skills, knowledge and competencies to address the local concerns. The resources that are available through the programme are then used to achieve the objectives of both parallel tracks. Strategies can be developed as part of the design to build skills and the time necessary to achieve this depends on the individual and collective capabilities of its members. Too short a programme timeframe runs the real risk of initiating community-level changes, only for the assistance to end before such changes have reached a satisfactory outcome.

Setting programme objectives

Objective setting within chronic disease prevention is usually centred on a reduction in morbidity and mortality 
and on lifestyle management such as a change in specific health-related behaviours. Objective setting for bottom-up approaches is centred on the level of control of the community over decisions regarding their health and its determinants. In parallel-tracking, the two sets of objectives are given equal emphasis and complement one another at each stage of the programme cycle.

In the example of the chronic disease prevention programme for a Polynesian community the bottom-up objectives include:

- To assist the community to establish vegetable gardens in 20 locations in South and West Auckland (area of high density of Polynesian peoples) before end of year XXXX;

- To assist the community to establish five walking groups for Polynesian men and women in South and West Auckland before end of year XXXX;

- To conduct 30 seminars on diabetes at pacific community centres in South and West Auckland before end of year XXXX.

The disease prevention objectives also need to be clear and achievable, for example, to:

- Reduce body mass index by $\mathrm{X} \%$ in $\mathrm{X} \%$ of the population before end of year XXXX;

- Bring blood pressure readings into normal range for $\mathrm{X} \%$ of the population before end of year XXXX;

- Bring cholesterol levels into normal range for X\% of the population before end of year XXXX.

Developing the strategic approach

The strategic approach of the programme typically uses the mass media but can also strengthen the bottom-up approach by systematically building community capacity. Allowing individuals to participate in groups that develop into community-based organisations centred on common interests and that later form partnerships can build capacity. The development of community organisations is crucial to allow individual members to make the transition to a broader network of alliances. It is through these partnerships that organisations are able to gain greater support and resources for their particular concerns. The key challenge is how practitioners structure their work with the intention to assist individuals to organise collectively to form stronger partnerships.

Programme management and implementation

The role of the programme management has traditionally been to control the process of planning, organising and implementing the disease prevention objectives. In bottom-up approaches, the role of the programme management is to be sympathetic to stakeholder ownership and involvement and to increasingly give control to the community. This can be achieved by encouraging the community to take increasing responsibility for the management of the programme and by building skills to enable them to contribute to the implementation. One way of developing skills is to be involved in short-term tasks that are realistic and achievable. To do this the practitioner can ask the community to set and achieve short-term goals. This is important because short-term successes can help to motivate people towards the achievement of long-term objectives. This would include setting up a demonstration vegetable garden and the provision of skills training to encourage others to start their own community gardens.

\section{Evaluation}

The final stage of 'parallel-tracking' is the evaluation of both the disease prevention and the bottom-up objectives of the programme. The bottom-up approach can be a long process and if measured solely as an outcome its achievements can be lost during the fixed timeframe of topdown programming. The evaluation of changes in the course of the process is, therefore, preferable as well as any particular outcome. The bottom-up typically uses participatory techniques and includes: the number of active vegetable gardens that were established; the number of active walking groups that were established and; the number of seminars completed. The top-down evaluation includes: the \% level of body mass; blood pressure and cholesterol levels reduced in the population group within a specified period of time.

\section{Conclusion}

The framework for parallel-tracking is designed to help clarify how bottom-up objectives can be systematically accommodated within top-down chronic disease prevention programmes. The framework is essentially a planning tool for determining activities that lead towards certain ends but can also be used to aid implementation. The framework is intended to be used by all stakeholders within a chronic disease prevention programme. Parallel-tracking requires a fundamental change in the way we think about programming and can have major benefits in resolving the tensions inherent between top-down and bottom-up approaches. This will be of interest to planners and practitioners who want to better accommodate community-based issues in programme design. 


\section{References}

Baum F (2007) Cracking the nut of health equity: top down and bottom up pressure for action on the social determinants of health. IUHPE promot educ 14(2):90-95

Knowler W, Barrett-Connor E, Fowler S, Hamman R, Lachin J, Walker E, Nathan D (2002) Reduction in the incidence of type 2 diabetes with lifestyle intervention or metformin. N Engl J Med 346:393-403

Laverack G (2004) Health promotion practice: power \& empowerment. London, SAGE Publications
Laverack G, Labonte R (2000) A planning framework for accommodation of community empowerment goals within health promotion programming. Health, Policy Plan 15(3):255-262

Ministry of Health and Pacific Island Affairs (2004) Tupu Ola Moui. Pacific health chart book. Ministry of Health, Wellington

Moana I (2005) Diabetes prevention in Tongan people. Project report common health 743. University of Auckland, Auckland (unpublished)

Tuomilehto J, Lindstrom J, Eriksson JG et al (2001) Prevention of type 2 diabetes mellitus by changes in lifestyle among subjects with impaired glucose tolerance. N Engl J Med 344:1343-1350 